SUPPLEMENTARY INFORMATION

\title{
Tunable long-range interactions between self- trapped beams driven by thermal response of photoresponsive hydrogels
}

Amos Meeks ${ }^{1}$, Rebecca Mac ${ }^{l}$, Simran Chathanat ${ }^{l}$, Joanna Aizenberg ${ }^{1,2 *}$

${ }^{1}$ John A. Paulson School of Engineering and Applied Sciences, Harvard University, Cambridge, MA 02138, USA

${ }^{2}$ Department of Chemistry and Chemical Biology, Harvard University, Cambridge, MA 02138, USA 


\section{Table of Contents}

\section{Section S1 Preparation and Characterization of Spiropyran-Modified Hydrogels}

S1.1 Materials

S1.2 Preparation of SP Gels

S1.2.1. Synthesis of Spiropyran Acrylate Monomer (1',3',3'-trimethylspiro[chromene-2,2'indolin]-6-yl acrylate)

S1.2.2. Synthesis of Hydrogels

S1.3 Characterization Methods of Spiropyran-Modified Hydrogels

S1.3.1 AFM Measurements of Gel Contraction

S1.3.2 Swelling Measurements as a Function of Temperature

Section S2 Thermal Effects in Single-Beam Self-Trapping

S2.1 Estimation of Local Temperature Increases During Self-Trapping

S2.2 Estimation of Effects of Temperature-Induced Deformation on Self-trapping

Section S3 Measurement of Poroelastic Diffusivity

Section S4 Interactions of Two Separated Self-trapped Beams

S4.1 Preparation of Bisected Gels

S4.2 Optical Assembly for Two Beam Self-trapping Experiments

S4.3 Scattering Cannot Explain Experimental Observations

Section S5 Descriptions of Supplemental Videos 


\section{S1. Preparation and Characterization of Spiropyran-Modified Hydrogels S1.1. Materials}

Acrylamide, acrylic acid, N,N'-methylenebis(acrylamide), N-isopropylacrylamide, dimethyl sulfoxide (DMSO), triethylamine (TEA), tetrahydrofuran (THF), 2-Hydroxy-4'-(2hydroxyethoxy)-2-methylpropiophenone, acryloyl chloride, trifluoroacetic acid (TFA), acetonitrile, ethyl acetate, sodium bicarbonate, (hydroxyethyl)methacrylate (HEMA), and hydrochloric acid were all purchased from MilliporeSigma (Missouri, USA). 1',3',3'-trimethyl6-hydroxyspiro(2H-1-benzopyran,2-2'-indoline) (Acros Organics, New Jersey, USA) was used without further purification.

\section{S1.2. Preparation of SP Gels}

S1.2.1. Synthesis of Spiropyran Acrylate Monomer (1',3',3'-trimethylspiro[chromene-2,2'indolin]-6-yl acrylate)

Spiropyran acrylate monomer (1',3',3'-trimethylspiro[chromene-2,2'-indolin]-6-yl acrylate) was synthesized based on a procedure described previously. ${ }^{1}$ Briefly, 1',3',3'-trimethyl-6hydroxyspiro(2H-1-benzopyran,2-2'-indoline) was treated with acryloyl chloride and triethylamine in THF at $0^{\circ} \mathrm{C}$ and reacted for $15 \mathrm{~h}$ at $25^{\circ} \mathrm{C}$. The product was washed in ethyl acetate with saturated aqueous sodium bicarbonate solution and brine. After the solvent was removed in a vacuum, ethyl acetate and a saturated sodium hydrogen carbonate aqueous solution were added, and the aqueous phase was extracted with ethyl acetate. The recovered organic phase was washed with brine, dried over anhydrous magnesium sulfate and filtered. After evaporation of the solvent, the residue was purified by silica gel column chromatography (1/6 ethyl acetate/n-hexane as an eluent) to obtain the acrylated spirobenzopyran monomer. Product purity was checked with H-NMR.

\section{S1.2.2. Synthesis of Hydrogels}

1:1 w/w acrylamide:acrylic acid was dissolved in 4:1 v/v DMSO:deionized water. To this solution, $2 \% \mathrm{w} / \mathrm{v} \mathrm{N}, \mathrm{N}$ '-methylenebis(acrylamide) was added as crosslinker. Acrylated spiropyran was dissolved in the pre-polymer solution at a concentration of $0.25 \mathrm{~mol} \%$ of total monomer. Upon addition of 2-Hydroxy-4'-(2-hydroxyethoxy)-2-methylpropiophenone as a photoinitiator, the hydrogel pre-polymer solution was dispensed into a circular aluminum mold (4 mm thick and $10 \mathrm{~mm}$ in diameter) sandwiched between two glass slides, and cured under a UV lamp (60 Hz, 3.5 A, 115 V, Blak-Ray, UVP, California, USA) at ambient temperature for 5 minutes on each side. Cured hydrogel samples were then immersed in deionized water. The water was replaced daily for 3 days to remove unreacted monomer and excess solvent. Finally, the hydrogel was transferred to a sample holder sandwiched between two glass slides containing deionized water for optical measurements. The sample holder consisted of a $3.8 \mathrm{~cm}$ x $3.8 \mathrm{~cm}$ x $0.6 \mathrm{~cm}$ aluminum block with a $1.3 \mathrm{~cm}$ diameter hole through its center. Gels containing N-isopropylacrylamide (NIPAAm) were prepared with 5\% w/v acrylic acid and dissolved in DMSO only. 


\section{S1.3 Characterization Methods of Spiropyran-Modified Hydrogels}

\section{S1.3.1 AFM Measurements of Gel Contraction}

The contraction of spiropyran-functionalized $\mathrm{p}(\mathrm{AAm}-\mathrm{co}-\mathrm{AAc})$ hydrogels under an intense, focused c.w. $532 \mathrm{~nm}$ laser was measured using a JPK Instruments NanoWizard 4a atomic force microscope (AFM) with the C cantilever of an All-In-One AFM probe bought from BudgetSensors. A $532 \mathrm{~nm}$ c.w. laser was collimated and shone into the camera port of a Leica DMI8 inverted optical microscope upon which the AFM device was placed. Line traces of the gel surface were taken over the site where the laser was focused, and the laser was switched off and on.

Total gel thickness was measured using an upright Zeiss LSM 700 confocal microscope by determining the height of the gel surface focal plane above the focal plane of its substrate, as demonstrated in Supplementary Figure S1. Linear contraction of the gel under laser light was then calculated by dividing the change in thickness of the gel under intense laser light by the total gel thickness.
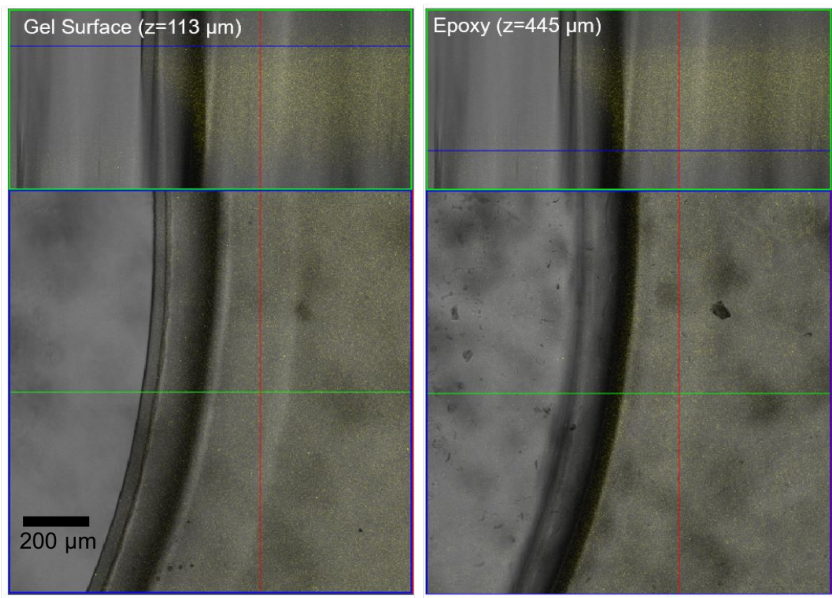

Figure S1 | Measurement of gel film thickness using optical and fluorescence microscopy.

\section{S1.3.2 Swelling Measurements as a Function of Temperature}

Pre-polymer solution was prepared according to the method in S1.2.2. A negative polydimethylsiloxane (PDMS) mold patterned with small (10-100s micron width) and thin $(<10$ microns) cylinders was prepared using a preexisting patterned silicon wafer. Thin samples were desired for their rapid equilibrium timescale, while regular shapes were desired to simplify length/radius measurements. A small volume $(\sim 6 \mu \mathrm{L})$ of pre-polymer solution was deposited onto the mold and covered by a glass slide. The solution was cured directly in the mold for 5 minutes under a UV lamp (60 Hz, 3.5 A, $115 \mathrm{~V}$, Blak-Ray, UVP, CA, USA). The mold was then immersed in deionized water to release the gel samples.

For all measurements, the temperature was manually adjusted using an Instec STC 200 temperature controller. During experiments, the gels were contained in a sample holder consisting of a custom $3.8 \mathrm{~cm}$ x $3.8 \mathrm{~cm}$ x $0.6 \mathrm{~cm}$ aluminum block with a $1.3 \mathrm{~cm}$ diameter hole 
through its center. The aluminum block was sandwiched between two glass slides adhered on each side of the hole with vacuum grease, thus creating a sealed, transparent well in which the samples could be deposited and equilibrated in deionized water. The sample holder was placed under a Zeiss LSM 700 confocal microscope on a stage connected to the temperature controller. The temperature was adjusted at regular intervals $\left(5^{\circ} \mathrm{C}\right)$. Corresponding images of the gel samples at each temperature setpoint were captured using a compatible software (ZEN Black 2011).

The radius measurements were obtained by first processing the image using a Gaussian filter, followed by applying Hough circle transform provided by the OpenCV Python library. The function performs edge detection, then determines a circle center and calculates a radius. For each image set, an appropriate set of parameters (minimum and maximum radius, distance between circles, edge intensity, etc.) was used. As these gels swell isotropically, the swelling

ratio is $S=\left[\frac{r(T)}{r\left(T_{0}\right)}\right]^{3}$, where $S$ is the swelling ratio, $r(T)$ is the radius at temperature $T$, and $r\left(T_{0}\right)$ is the radius at the initial temperature.

\section{Section S2 Thermal Effects in Self-Trapping}

\section{S2.1 Estimation of Local Temperature Increases During Self-Trapping}

Direct measurement of the local temperature changes is difficult due to the small area and the inaccessibility of the beam path within the bulk hydrogel sample. Instead we estimate the local temperature changes using analytical and numerical methods. We model the heat transport in cylindrical coordinates as diffusive with a heat source that is dependent on the local intensity:

$$
\frac{\partial u(r, z, t)}{\partial t}=\frac{k}{\rho c_{P}}\left(\frac{1}{r} \frac{\partial u(r, z, t)}{\partial r}+\frac{\partial^{2} u(r, z, t)}{\partial r^{2}}+\frac{\partial^{2} u(r, z, t)}{\partial z^{2}}\right)+\frac{2 \alpha}{\rho c_{P}} I(r, z, t)
$$

Where $u(r, z, t)$ is the temperature as a function of radial distance from the beam distance along the beam path, and time. $k$ is the thermal conductivity, which was measured to be approximately $0.4 \mathrm{~W} \mathrm{~m}^{-1} \mathrm{~K}^{-1}$ for similar gels ${ }^{2} ; \rho$ is the density of water, $1000 \mathrm{~kg} \mathrm{~m}^{-3} ; c_{P}$ is the heat capacity of water, $4,182 \mathrm{~J} \mathrm{~kg}^{-1} \mathrm{~K}^{-1}$; and $\alpha$ is the absorption coefficient, which was previously estimated to be about $800 \mathrm{~m}^{-1}$ (ref 3$) . I(r, z, t)$ is the intensity of the beam as a function of radial distance, distance along the beam path, and time. For the purpose of estimation, we assume that the beam is fully self-trapped throughout the gel (its width does not change with $z$ ) and that it is constant in time. The beam's initial input intensity $I_{0}$ is determined by the beam power and the beam intensity decreases exponentially due to absorption, leading to the expression for the intensity:

$$
I(r, z)=I_{0} e^{-\frac{r^{2}}{w^{2}}} e^{-\alpha z}
$$

Where $w$ is the constant beam width, which we assume is $20 \mu \mathrm{m}$, and outside of the appropriate $z$ boundaries of the gel we assume that $I=0$. On the front and back sides of the gel we model a $1 \mathrm{~mm}$ thick piece of glass, with a thermal conductivity of $1 \mathrm{~W} \mathrm{~m}^{-1} \mathrm{~K}^{-1}$. Fixed temperature boundary conditions were used. 
This problem was solved using Mathematica's built-in finite-element solving methods. The simulation results are shown in Figure S2. The maximum simulated temperature increase was about $3.6 \mathrm{~K}$.

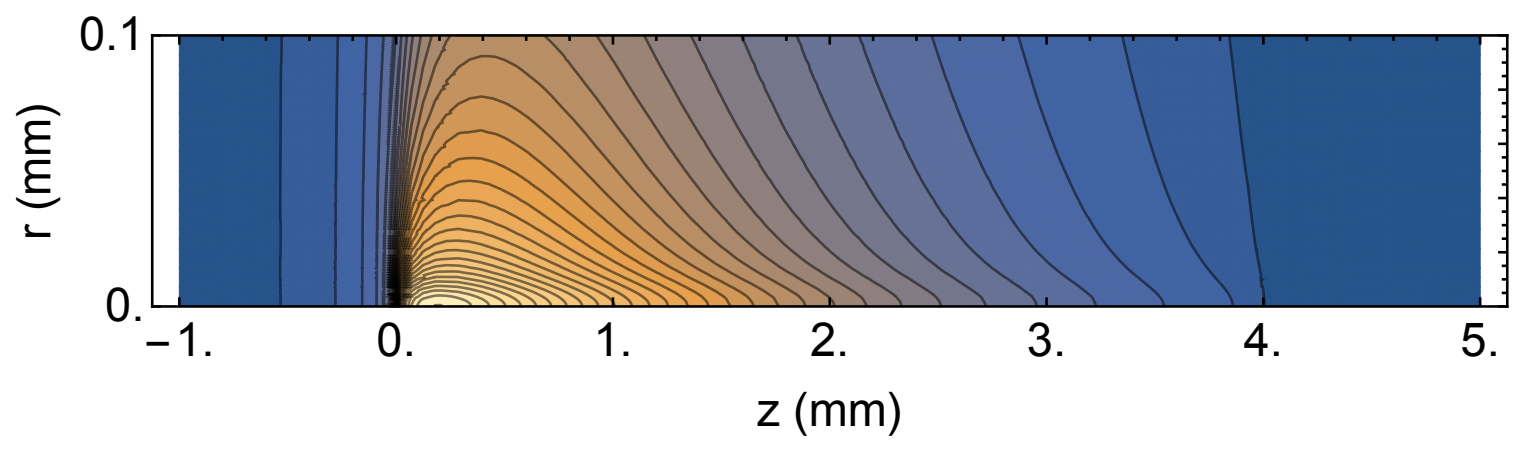
0.4
0.8
1.2
1.6
2.0
2.4
2.8
3.2

Figure S2 | Finite Element Modeling results of the steady state temperature increase in degrees K due to a self-trapped 20 $\boldsymbol{\mu m}$ wide, $6 \mathbf{~ m W}$ c.w. beam. The gel domain runs from $\mathrm{z}=0$ to $\mathrm{z}=4 \mathrm{~mm}$, with $1 \mathrm{~mm}$ of glass on both ends. Temperature difference is fixed to zero at the boundaries. The maximum temperature reached occurs at about $\mathrm{z}=0.2 \mathrm{~mm}$ and corresponds to an increase of about $3.6 \mathrm{~K}$.

\section{S2.2 Estimation of Effects of Temperature-Induced Deformation on Self-trapping}

Changes in temperature affect the propagation of light through the thermal swelling/deswelling of the hydrogel. The changes in swelling as a function of temperature measured in Figure $2 \mathrm{c}$ and $2 \mathrm{~d}$ are related to the engineering strain $e$ by

$$
e=\left(\frac{V}{V_{0}}\right)^{\frac{1}{3}}-1
$$

Using this we measure, near room temperature, an engineering strain of $0.43 \% / \mathrm{K}$ for $\mathrm{p}(\mathrm{AAm}-$ co-AAc) hydrogels and $-0.54 \% / \mathrm{K}$ for $\mathrm{p}$ (NIPAAm-co-AAc) hydrogels. The effect of strain on the refractive index has been described in detail previously ${ }^{3}$ and is briefly summarized here. In the linear approximation the volume fraction $\phi$ is related to the strain as $\phi=\phi_{e q}(1-3 e)$. We can define a dimensionless change in the volume fraction of polymer $\psi=\frac{\phi-\phi_{e q}}{\phi_{e q}}=-3 e$. The dimensionless change in the volume fraction of the polymer is then related to the refractive index change $\Delta n$ by $\Delta n=\left(n_{p}-n_{s}\right) \phi_{e q} \psi$ where $n_{p}$ is the refractive index of the polymer phase and $n_{s}$ is the refractive index of the solvent phase. Thus $\Delta n=-3\left(n_{p}-n_{s}\right) \phi_{e q} e$. Using $n_{p}=1.49$, $n_{s}=1.33$, and $\phi_{e q}=0.4$ we get a change with temperature of $-0.0008 / \mathrm{K}$ for $\mathrm{p}$ (AAm-co-AAc) and $0.001 / \mathrm{K}$ for $\mathrm{p}$ (NIPAAm-co-AAc).

What matters for light propagation is not the absolute change in refractive index, but rather the local gradient. Thus, while the absolute change of around $4 \mathrm{~K}$ near the beam center is fairly large, the temperature difference one beam radius $(10 \mu \mathrm{m})$ away from the beam center is only about $0.5 \mathrm{~K}$. This leads to a local refractive index contrast that is smaller than but still 
comparable to the $\Delta n$ due to spiropyran isomerization, suggesting that temperature effects likely play some role in single beam self-trapping.

The situation is different for two beams. In this case the rotational symmetry is broken, so even the small temperature gradient seen across the beam as a result of the other beam can lead to measurable bending of the beam. The computations from Section S2.1 suggest that at a distance about $200 \mu \mathrm{m}$ away from the beam center, the average temperature gradient over $20 \mu \mathrm{m}$ is about $0.025 \mathrm{~K}$, corresponding in $\mathrm{p}$ (AAm-co-AAc) to a refractive index gradient of about $2 \times 10^{-5}$, or about $1 \mathrm{~m}^{-1}$. We can estimate the beam bending that would result from this by considering the ray tracing equation:

$$
\frac{d}{d s}\left[n(\vec{r}) \frac{d \vec{r}}{d s}\right]=\nabla n(\vec{r})
$$

Where $\vec{r}$ is the beam path, $n$ is the spatially varying refractive index, and $\frac{d}{d s}$ is the line derivative along the beam. We assume that the beam begins propagating directly along the $x$ axis and that $n$ varies only along the $y$ direction. Noting that, if $\theta$ is the angle between the beam direction and the $x$ axis, $\tan \theta=\frac{d y}{d x}$, the equation then becomes

$$
\frac{d^{2} r_{y}(x)}{d x^{2}}=\frac{n^{\prime}(y)}{n(y)}\left(\tan ^{2} \theta(x)+1\right)
$$

Where $r_{y}(x)$ is the $y$ position of the beam as a function of $x$. Given that the beam bends by a small amount over the distances considered, we can assume that $\tan ^{2} \theta(x) \ll 1$, which simplifies the problem to that of a particle under a constant acceleration of $\frac{n^{\prime}(y)}{n(y)}$. The solution is thus $r_{y}(x)=\frac{n^{\prime}(y)}{2 n(y)} x^{2}$. As mentioned previously, as a distance of about $200 \mu \mathrm{m}$ from the beam center, $n^{\prime}(y) \approx 1 \mathrm{~m}^{-1}$, and $n \approx 1.49$. For a propagation distance of $4 \mathrm{~mm}$ this gives an approximate deflection in the $y$ direction of about $5.4 \mu \mathrm{m}$, which corresponds very well to the observed deflections shown in Figure 3c.

\section{Section S3 Measurement of Poroelastic Diffusivity}

The poroelastic diffusivity of the spiropyran-modified $\mathrm{p}$ (AAm-co-AAc) hydrogels was measured using the method described in $\mathrm{Hu}$ et al. ${ }^{4}$ Briefly, this method involves indenting a bulk hydrogel sample immersed in water and measuring the force relaxation over time. We used a cylindrical punch of radius $559 \mu \mathrm{m}$. For a cylindrical punch the given empirical formula used to solve for the poroelastic diffusivity $D$ is:

$$
\frac{F(t)-F(\infty)}{F(0)-F(\infty)}=1.304 e^{-\sqrt{\frac{D t}{a^{2}}}}-0.304 e^{-0.254 \frac{D t}{a^{2}}}
$$


Where $F(t)$ is the force as a function of time $t$, and $a$ is the radius of the cylindrical punch. We fit equation (6) to the force measurement data (Figure S3) with $D$ and $F(\infty)$ as free parameters using Mathematica version 11.3.0.0's built in NonlinearModelFit function. The best fit value of $D$ was found to be $1.8 \times 10^{-11} \mathrm{~m}^{2} \mathrm{~s}^{-1}$, and the fit is shown in Figure $\mathrm{S} 3$.

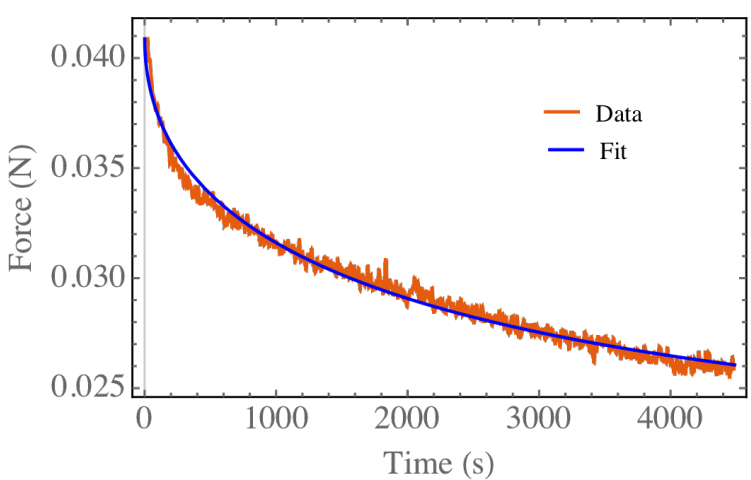

Figure S3 | Measurement of poroelastic diffusivity. Force over time for a constant indentation with a cylindrical punch of radius $559 \mu \mathrm{m}$. The blue line shows a fit of equation (6).

\section{Section S4 Interactions of Two Separated Self-trapped Beams}

\section{S4.1 Preparation of Bisected Gels}

Gels were prepared as described in S1.2.2 except that the aluminum ring mold was cut into two pieces. Between the two halves of the ring a $4 \mathrm{~mm}$ by $12 \mathrm{~mm}$ piece of \#0 glass was sandwiched with a small amount of silicone vacuum grease used to hold the assembly together. This mold was then filled with prepolymer solution and sandwiched between two glass slides before being cured under a mercury UV lamp. After curing the sandwiching glass slides were removed and the entire ring assembly with glass divider was immersed in water for three days with the water being replaced every 24 hours. After equilibration the gel shrinks slightly, and so a folded aluminum spacer is inserted to keep the two halves of the gel pressed against the glass divider.

\section{S4.2 Optical Assembly for Self-trapping Experiments}

The optical assembly for single beam self-trapping experiments is shown in Figure S4a. A continuous wave diode-pumped solid-state laser (Thorlabs, Inc., New Jersey USA) was used as the excitation source. The beam $(\lambda=532 \mathrm{~nm})$ was collimated $(\mathrm{L} 1, \mathrm{f}=2.54 \mathrm{~cm})$ before passing through a $\lambda / 2$ waveplate $(\mathrm{W})$ and a polarizing beam splitter cube $(\mathrm{C})$. It was then passed through an absorption filter $(\mathrm{F})$ and then through a focusing lens $(\mathrm{L} 2, \mathrm{f}=2.54 \mathrm{~cm})$. This produced a beam focused to a diameter of $\sim 20 \mu \mathrm{m}$ onto the entrance face of a hydrogel sample within the sample cell (S). The profile of the beam at the exit face of the sample was imaged by a pair of planoconvex lenses $(\mathrm{L} 3, \mathrm{f}=25.4 \mathrm{~mm} ; \mathrm{L} 4, \mathrm{f}=25.4 \mathrm{~mm}$; total magnification $=20 \mathrm{x})$ onto a charge-coupled device (CCD) camera (1200 (H) x $1600(\mathrm{~W})$ pixels, $7.4 \mu \mathrm{m}(\mathrm{H})$ x $7.4 \mu \mathrm{m}(\mathrm{W})$ pixel size; Retiga-2000R Color Cooled, QImaging, British Columbia, Canada). The camera was operated using the open source $\mu$ Manager software, and images were analyzed using Python and 
Mathematica. As beams self-trap their peak intensity increases significantly. Saturation was avoided by changing the exposure time of the camera during acquisition. Changes in exposure time were recorded and corrected for after acquisition. In all self-trapping experiments, the beam diameter was measured at the exit face of the sample. Beam radius was calculated by counting the number of pixels with intensity greater than $1 / \mathrm{e}^{2}$ of the peak intensity and then calculating $\sqrt{A / \pi}$ where $A$ is the area corresponding to the number of pixels.

Double beam self-trapping experiments were carried out on an optical assembly similar to the single beam setup, but with the introduction of a second beam parallel to the first beam (Figure S4b). The output beam was passed through a $\lambda / 2$ waveplate (W) and split into two beams using a polarizing beam splitter cube (C). The $\lambda / 2$ waveplate orientation was adjusted to change the ratio of intensities between the two beams. The beams were directed by mirrors (M) and a second polarizing beam splitter cube, through a set of filters (F) and a biconvex lens (L2, $f=2.54$ $\mathrm{cm}$ ), which focused the beams to a diameter of $20 \mu \mathrm{m}$ onto the entrance face of the sample in the sample cell. The separation distance between the focused beams could be adjusted using a linear stage to change the relative position of one of the mirrors. The result was two orthogonally polarized beams of light with variable separation at the entrance of the sample.

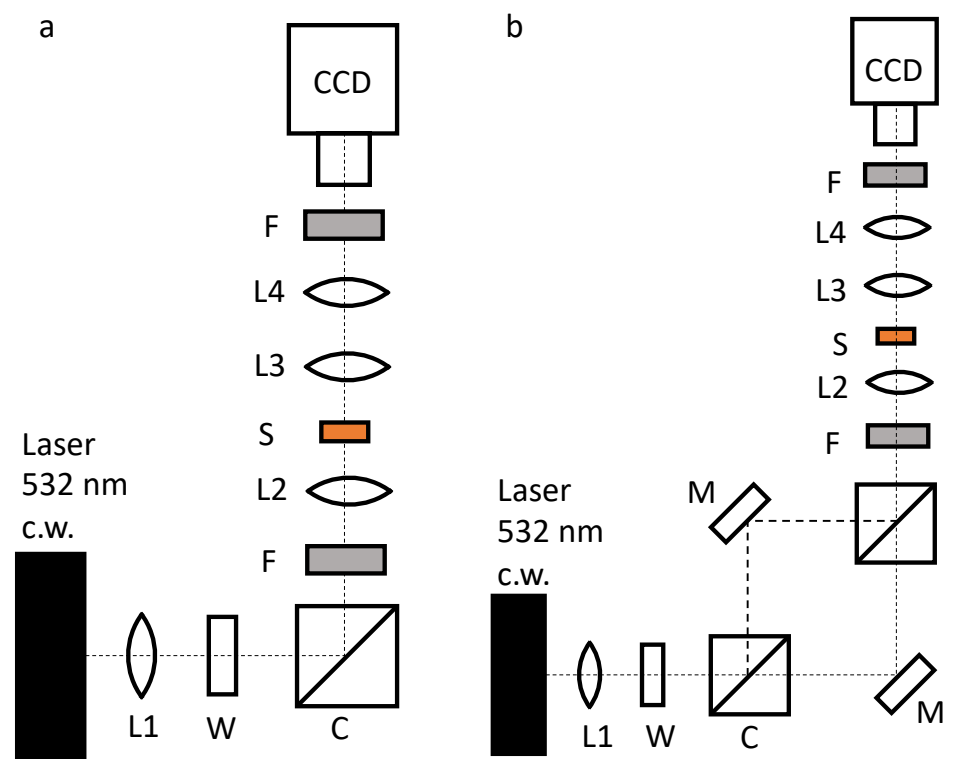

Figure S4 | Optical setup for self-trapping experiments. a) Single beam optical self-trapping experiment including the sample (S), a $\lambda / 2$ wave plate (W), polarizing beam splitter cube (C), absorption filters (F), biconvex lenses (L1-L4) and a CCD camera.

b) Double-beam optical self-trapping experiment with additional beam splitter cube and mirrors (M).

\section{S4.3 Scattering Cannot Explain Experimental Observations}

We show here that long-range interactions through scattered light cannot explain the experimental observations. This is clear from three different arguments: 1) the intensity of scattered light is too low to create significant interactions on a reasonable timescale; 2) optical interactions should lead to attractive interactions, contrary to the observed repulsive interactions; 
and 3 ) scattered light interactions would be expected to be stronger in the less absorbing p(NIPAAm-co-AAc) hydrogels, while observed interactions are weaker in these gels.

First, we consider the intensity of scattered light. We assume that scattering is uniform from the cylindrical beam and find that

$$
\frac{I(R)}{I_{0}}=\frac{\alpha w^{2}}{2 R} \approx 1 \times 10^{-4}
$$

Where $I(R)$ is the intensity of scattered light at a distance $R$ away from the beam, $I_{0}$ is the peak intensity of the beam from which light is scattering, $w$ is the width of the cylindrical beam, and $\alpha$ is the scattering coefficient. We can get a high estimate for the intensity of the scattered light by assuming that all power loss as the beam propagates through the gel is due to scattering. The loss coefficient was measured by Morim, Meeks, Shastri et al. ${ }^{3}$ to be about $800 \mathrm{~m}^{-1}$. Using this value for $\alpha$, a beam width of $20 \mu \mathrm{m}$, and a distance $R$ of $200 \mu \mathrm{m}$ we get an intensity ratio on the order of $1 \times 10^{-4}$. Noting that the timescale of self-trapping dynamics are inversely proportional to light intensity, and that self-trapping occurs on a timescale of $\sim 10^{1} \mathrm{~s}$, this suggests that beam interactions mediated by scattered light would occur with a timescale of $\sim 10^{5} \mathrm{~s}$, which is not consistent with the observed large and fast interactions.

Second, we point out that light causes the gel to contract, which increases the refractive index. Thus, any scattered light from a co-propagating beam should contract the gel more in the direction of the beam, which would bend the beams towards each other and lead to an attractive interaction. This is at odds with the observed repulsive interactions.

Finally, we consider that, in addition to scattering, these gels absorb green light. Specifically, the $\mathrm{p}$ (AAm-co-AAc) hydrogels can be seen by eye to be redder than the p(NIPAAm-co-AAc) hydrogels, suggesting that they absorb more strongly in the green region of the spectrum. If these gels absorb more strongly, and if scattered light plays a role in beam interactions, then it would be expected that beam interactions would be stronger in the p(NIPAAm-co-AAc) gel compared to the $\mathrm{p}(\mathrm{AAm}-\mathrm{co}-\mathrm{AAc})$ gel. However, the opposite trend is observed, which shows that scattered light cannot play a dominant role in long-range beam interactions.

\section{Section S5 Descriptions of Supplemental Videos}

MOVIE_S1

Video of long-range two beam interactions in a spiropyran-functionalized $\mathrm{p}$ (AAm-co-AAc) hydrogel, sped up 80x. Scale bar is $100 \mu \mathrm{m}$.

\section{MOVIE_S2}

Video of long-range two beam interactions in a spiropyran-functionalized $\mathrm{p}$ (AAm-co-AAc) hydrogel that is bisected by a $\sim 100 \mu \mathrm{m}$ glass coverslip, sped up 40x. Scale bar is $100 \mu \mathrm{m}$.

MOVIE_S3 
Video of long-range two beam interactions in a spiropyran-functionalized p(NIPAAm-co-AAc) hydrogel, sped up 80x. Scale bar is $100 \mu \mathrm{m}$.

\section{MOVIE_S4}

Video of short-range two beam interactions in a spiropyran-functionalized p(NIPAAm-co-AAc) hydrogel showing spiraling and merging, sped up 80x. Scale bar is $100 \mu \mathrm{m}$.

\section{References}

1. Sugiura, S. et al. On-demand microfluidic control by micropatterned light irradiation of a photoresponsive hydrogel sheet. Lab Chip 9, 196-198 (2009).

2. Hirata, Y., Kato, Y., Andoh, N., Fujiwara, N. \& Ito, R. Measurements of thermophysical properties of polyacrylamide gel used for electrophoresis. J. Chem. Eng. JAPAN 26, 143147 (1993).

3. Morim, D. R. et al. Opto-chemo-mechanical transduction in photoresponsive gels elicits switchable self-trapped beams with remote interactions. Proc. Natl. Acad. Sci. U. S. A. 117, 3953-3959 (2020).

4. Hu, Y., Zhao, X., Vlassak, J. J. \& Suo, Z. Using indentation to characterize the poroelasticity of gels. Appl. Phys. Lett. 96, 121904 (2010). 